\title{
Minimax design of digital FIR filters using linear programming in bandwidth interleaving digital-to-analog converter
}

\author{
Xing Yang, Houjun Wang, Ke Liu ${ }^{\text {a) }}$, Yindong Xiao, Zaiming Fu, \\ and Guangkun Guo
}

School of Automation Engineering, University of

Electronic Science and Technology of China,

Chengdu 611731, China

a)liuke@uestc.edu.cn

\begin{abstract}
Bandwidth interleaving digital-to-analog converter (BI-DAC) is a new method for breaking through the bandwidth restriction of the DAC to generate a wideband signal. However, there are some errors in the BI-DAC system such as the aliasing errors caused by the non-ideal performance of the analog filters. To achieve the aliasing errors cancellation, this paper studied the minimax design of digital finite impulse response (FIR) filters. The design goal was to meet a given desired spurious free dynamic range (SFDR) of the BI-DAC system. The problem of designing the digital FIR filters was formulated as a linear programming (LP) problem which could be used to find the global optimal solution of the coefficients of the digital FIR filters. Additionally, this proposed design method performance analysis consist of the computational complexity was derived. Finally, all the proposed designs are verified by both theoretical analysis and numerical simulations, and satisfactory simulation results were achieved.
\end{abstract}

Keywords: bandwidth interleaving, linear programming, minimax design, aliasing errors cancellation, digital FIR filters

Classification: Circuits and modules for electronic instrumentation

\section{References}

[1] X. Yang, et al.: "Estimation and compensation methods of time delay and phase offset in hybrid filter bank DACs," Electron. Lett. (accepted manuscript) (DOI: 10.1049/el.2018.0937).

[2] X. Chen, et al.: "All-electronic 100-GHz bandwidth digital-to-analog converter generating PAM signals up to 190 GBaud," J. Lightwave Technol. 35 (2017) 411 (DOI: 10.1109/JLT.2016.2614126).

[3] C. Schmidt, et al.: "Enhancing the bandwidth of DACs by analog bandwidth interleaving," Broadband Coverage in Germany, 10. ITG-Symposium (2016) 1. multiplexing," International Conference on OFDM and Frequency Domain 
Techniques, ICOFDT (2016) 1.

[5] C. Schmidt, et al.: "Digital signal splitting among multiple DACs for analog bandwidth interleaving (ABI)," IEEE International Conference on Electronics, Circuits and Systems, ICECS (2016) 245 (DOI: 10.1109/ICECS.2016. 7841178).

[6] T. B. Deng: "Decoupling minimax design of low-complexity variable fractional-delay FIR digital filters," IEEE Trans. Circuits Syst. I, Reg. Papers 58 (2011) 2398 (DOI: 10.1109/TCSI.2011.2123510).

[7] K. J. Kim and S. W. Nam: "FIR filter solution to unusual gain responses arising in Parks-McClellan algrithm," IEICE Electron. Express 6 (2009) 1226 (DOI: 10.1587/elex.6.1226).

[8] Y. Zhao, et al.: "Model calibration of non-ideal lowpass filter in modulated wideband converter for compressive sampling," COMPEL 34 (2015) 941 (DOI: 10.1108/COMPEL-06-2014-0138).

[9] A. Aggarwal, et al.: "Optimal design of 2D FIR filters with quadrantally symmetric properties using fractional derivative constraints," Circuits Syst. Signal Process. 35 (2016) 2213 (DOI: 10.1007/s00034-016-0283-x).

[10] J. Song, et al.: "Digital correction of frequency-response errors in bandwidthinterleaved ADCs," Electron. Lett. 52 (2016) 1596 (DOI: 10.1049/el.2016. 2433).

[11] K. Liu, et al.: "Precisely synchronous and cascadable multi-channel arbitrary waveform generator," Rev. Sci. Instrum. 88 (2017) 035110 (DOI: 10.1063/ 1.4978067).

[12] J. Zhang, et al.: “A 2.5-GHz direct digital frequency synthesizer with spurious noise cancellation,” IEICE Electron. Express 11 (2014) 20140533 (DOI: 10. 1587/elex.11.20140533).

[13] J. F. Sturm: "Using SeDuMi 1.02, a MATLAB toolbox for optimization over symmetric cones,” Optim. Methods Softw. 11 (1999) 625 (DOI: 10.1080/ 10556789908805766).

[14] R. H. Walden: “Analog-to-digital converter survey and analysis," IEEE J. Sel. Areas Commun. 17 (1999) 539 (DOI: 10.1109/49.761034).

\section{Introduction}

Bandwidth interleaving digital-to-analog (BI-DAC) $[1,2,3,4,5]$ is an emerging method for breaking through the bandwidth limitation of digital-to-analog converter (DAC) and increasing the available signal bandwidth. However, in the BIDAC system, the wideband output can be distorted due to the aliasing errors caused by the non-ideal analog filters and mixers performance. Non-ideal mixers performance was compensated by the digital pre-distortion technique, but this paper did not identify the method for compensating the non-ideal analog filters performance [2]. Non-ideal filters performance could be compensated by pre-distorting the input digital signal with the calibration matrix [3], or by designing the digital filters [4], and yet these methods either have heavy computation requirements, or they need additional digital filters, increasing the implementation complexity of the BI-DAC system.

It well known that, the aliasing errors caused by the non-ideal analog filters 
the BI-DAC system. Recently, the digital FIR filters optimal design methods have been the subject of active research $[6,7,8,9,10]$. In [6], the high-accuracy lowcomplexity FIR variable fractional delay (VFD) digital filters using linear programming (LP) in the minimax error sense, was proposed to minimize the maximum absolute error of the variable frequency response (VFR) of an FIR VFD filter. This paper did not mention that how to realize the digital FIR filters optimal design in the BI-DAC system, but it had its advantages and provided the reference for the digital FIR filters optimal design in the BI-DAC system. In the bandwidth interleaving analog-to-digital converter (BI-ADC) system which is the inverse system of BI-DAC, the digital FIR filters design using the biconjugate gradient stablished algorithm was proposed for canceling the aliasing errors caused by the non-ideal analog filters performance [10]. It well known that, if one of the digital and analog filter banks is designed firstly and fixed, the other filter bank can be formulated as an optimization problem. Therefore, the digital FIR filters design method proposed in [10], is also applicable for the BI-DAC system. But comparing with the LP technique, this method can achieve a larger aliasing errors and computational complexity.

This paper proposed a new digital FIR filters design using LP for the BI-DAC system. In this design method, Butterworth filters were chosen as the analog analysis filters. Given the analog analysis filters, the digital FIR filters were chosen for splitting the digital wideband signal into multiple sub-bands. The aliasing errors can be canceled by designing these digital FIR filters. The problem of designing these digital FIR filters was formulated as an optimization problem and solved using LP. As an optimization problem, if the global optimal solution exists, LP can be used to find it. Further, this design method can be proposed under the minimax criteria. This is very important because the aliasing errors can be markedly lower and improve the accuracy of the BI-DAC system by controlling the spurious free dynamic range (SFDR). The SFDR, which is equal to the ratio of the root mean square (RMS) amplitude of the maximum signal component to the RMS value of the next-highest distortion component, is a significant measure of the DAC accuracy $[11,12]$.

The main contributions of this paper are summarized as follows

1) In the specific context of BI-DAC, we proposed the digital FIR filters design using LP under the minimax criteria for canceling the aliasing errors caused by the non-ideal analog filters performance.

2) We compared the effectiveness and the computational complexity of the optimation design method in [10] and the minimax design using LP proposed in this paper. It was verified that our proposed design method had a better aliasing errors cancellation and much lower computational complexity than the optimation design method in [10].

The rest of this paper is organized as follows. The principle of the BI-DAC system is illustrated in Section 2. Section 3 gives the analog analysis filters design, as well as the minimax design of the digital FIR filters using LP. In Section 4, the effectiveness and the computational complexity of this proposed design method are evaluated and analyzed by different simulations. Ultimately, conclusions are summarized in Section 5. 


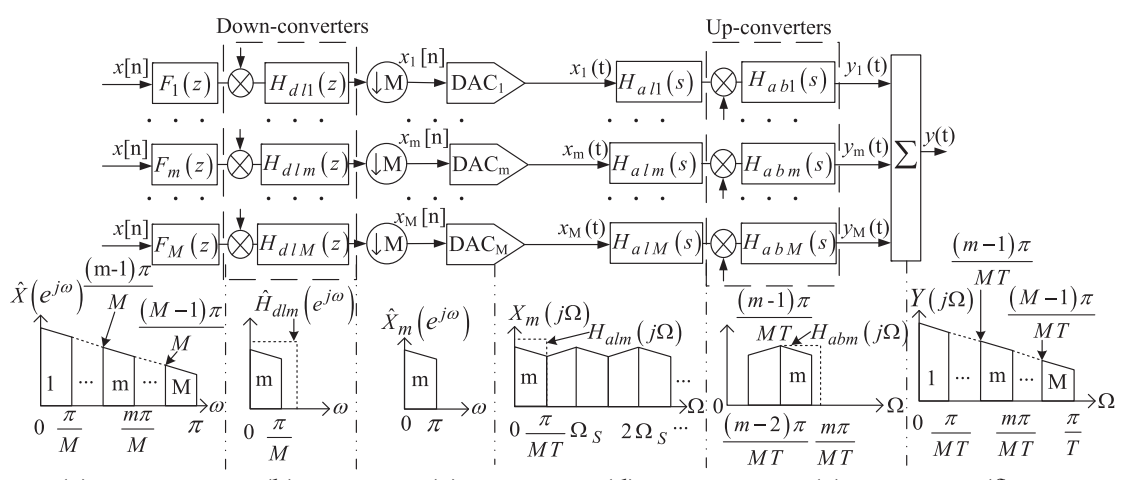

(a)

(b)

(d)

(e)

(f)
Fig. 1. Structure diagram of an $M$-channel BI-DAC.

\section{Principle of BI-DAC}

An $M$-channel BI-DAC is shown in Fig. 1, where the sampling rate and the bandwidth of the BI-DAC system are, respectively, $\frac{1}{T}$ and $\frac{\pi}{T}$. In the $m$ th channel, as shown in Fig. 1(a)-(c), the digital FIR filter $\hat{F}_{m}\left(e^{j \omega}\right)$ is used to split the input digital wideband signal $\hat{X}\left(e^{j \omega}\right)$ into a digital sub-band signal which is then downconvertered to the baseband. The unwanted sideband of the digital baseband signal is filtered out using the digital low pass filter $\hat{H}_{d l m}\left(e^{j \omega}\right)$, and then is down-sampled to generate the $\mathrm{DAC}_{m}$ input $\hat{X}_{m}\left(e^{j \omega}\right)$. So the $\mathrm{DAC}_{m}$ output is given by

$$
\begin{aligned}
X_{m}(j \Omega)= & \frac{1}{M T} \sum_{k=-\infty}^{+\infty} \hat{X}_{m}\left(e^{j(\Omega M T-2 \pi k)}\right) H(j \Omega) \\
= & \frac{1}{M T} \sum_{k=-\infty}^{+\infty} \hat{X}\left(e^{j\left(\Omega T-\frac{2 \pi k}{M}\right)+\frac{(m-1)}{M} \pi}\right) H(j \Omega) \\
& \times \hat{F}_{m}\left(e^{j\left(\Omega T-\frac{2 \pi k}{M}\right)+\frac{(m-1)}{M} \pi}\right) \hat{H}_{d l m}\left(e^{j\left(\Omega T-\frac{2 \pi k}{M}\right)}\right),
\end{aligned}
$$

where $\omega=\Omega M T$, and $H(j \Omega)$ is the frequency response of the preserving function of the DAC. As shown in Fig. 1(d), the images in $X_{m}(j \Omega)$ are located at the center frequency $k \Omega_{S}$, where $k$ is contributed from (1) and $\Omega_{S}=\frac{2 \pi}{M T}$. Then, as shown in Fig. 1(e), these images are filtered out by the analog low pass filter $H_{a l m}(j \Omega)$. The baseband analog signal is up-convertered to the high-frequency band, which is the sum of the unwanted and wanted sidebands. After canceling the unwanted sideband using the analog bandpass filter $H_{a b m}(j \Omega)$, the $m$ th channel output $Y_{m}(j \Omega)$ is given by

$$
\begin{aligned}
Y_{m}(j \Omega)= & \frac{1}{M T} \sum_{k=-\infty}^{+\infty} \hat{X}\left(e^{j\left(\Omega T-\frac{2 \pi k}{M}\right)}\right) \hat{F}_{m}\left(e^{j\left(\Omega T-\frac{2 \pi k}{M}\right)}\right) \\
& \times \hat{H}_{d l m}\left(e^{j\left(\Omega T-\frac{2 \pi k}{M}\right)-\frac{(m-1)}{M} \pi}\right) H\left(j\left(\Omega-\frac{(m-1)}{M T} \pi\right)\right) \\
& \times H_{a l m}\left(j\left(\Omega-\frac{(m-1)}{M T} \pi\right)\right) H_{a b m}(j \Omega) .
\end{aligned}
$$

Finally, all of the outputs $Y_{m}(j \Omega), m=1, \ldots, M$ are summed together to yield the analog wideband signal $Y(j \Omega)$ with $\Omega \in\left[0, \frac{\pi}{T}\right]$, which is given by 


$$
\begin{aligned}
Y(j \Omega)= & \sum_{m=1}^{M} Y_{m}(j \Omega) \\
= & \frac{1}{M T} \sum_{m=1}^{M} \sum_{k=-\infty}^{+\infty} \hat{X}\left(e^{j\left(\Omega T-\frac{2 \pi k}{M}\right)}\right) \hat{F}_{m}\left(e^{j\left(\Omega T-\frac{2 \pi k}{M}\right)}\right) \\
& \times \hat{H}_{d l m}\left(e^{j\left(\Omega T-\frac{2 \pi k}{M}\right)-\frac{(m-1)}{M} \pi}\right) H\left(j\left(\Omega-\frac{(m-1)}{M T} \pi\right)\right) \\
& \times H_{a l m}\left(j\left(\Omega-\frac{(m-1)}{M T} \pi\right)\right) H_{a b m}(j \Omega) .
\end{aligned}
$$

As shown in Fig. 1(f), the bandwidth of $Y(j \Omega)$ is $M$ times as that of $Y_{m}(j \Omega)$, $m=1, \ldots, M$. Consequently, the bandwidth limitation of each sub-DAC can be broken through in the BI-DAC system, which is the same as the description in Section 1.

\section{Filters design in the BI-DAC system}

In this Section, we mainly describe the designs of the analog analysis filters and the digital FIR filters. Further, we also analyze the computational complexity of our proposed design method.

\subsection{Analog analysis filters design}

In the BI-DAC system, high-performance wideband signals require that analog analysis filters not have constant group delay and passband ripples. Considering that Butterworth filters have stable passband magnitude response without constant group delay and passband ripples, second-order Butterworth filters were chosen as the analog analysis filters due to the generality of the BI-DAC system design, and they were used to eliminate the images in the DAC output and the unwanted bands generated by the up-conversion, respectively.

\subsection{Digital FIR filters design}

In (3), we only remain the terms with $k=0, \ldots, M-1$ so that (3) can be rewritten as

$$
Y(j \Omega)=\sum_{k=0}^{M-1} \hat{X}\left(e^{j\left(\Omega T-\frac{2 \pi k}{M}\right)}\right) T_{k}(j \Omega),
$$

where

$$
\begin{aligned}
T_{k}(j \Omega)= & \frac{1}{M T} \sum_{m=1}^{M} \hat{F}_{m}\left(e^{j\left(\Omega T-\frac{2 \pi k}{M}\right)}\right) \\
& \times \hat{H}_{d l m}\left(e^{j\left(\Omega T-\frac{2 \pi k}{M}\right)-\frac{(m-1)}{M} \pi}\right) H\left(j\left(\Omega-\frac{(m-1)}{M T} \pi\right)\right) \\
& \times H_{a l m}\left(j\left(\Omega-\frac{(m-1)}{M T} \pi\right)\right) H_{a b m}(j \Omega),
\end{aligned}
$$

and $T_{k}(j \Omega), k=0,1, \ldots, M-1$ denote the real transfer function of the BI-DAC system. It can be seen from (4) that the wideband signal is a mixture of the input signals frequency-shifted versions. The distortion function $T_{0}(j \Omega)$ denotes the 
group delay and the magnitude gain of the BI-DAC system. The aliasing function $T_{k}(j \Omega)$ for $k=1, \ldots, M-1$ denote the unwanted and shifted versions of the input signal that should be canceled. In the BI-DAC system, $F_{m}(z)$ denotes an N-tap digital FIR filter with $z$-transform as follows

$$
F_{m}(z)=\sum_{n=0}^{N-1} f_{m}(n) z^{-n}
$$

Hence, the frequency response $\hat{F}_{m}\left(e^{j\left(\Omega T-\frac{2 \pi k}{M}\right)}\right)$ can be expressed as

$$
\hat{F}_{m}\left(e^{j\left(\Omega T-\frac{2 \pi k}{M}\right)}\right)=\sum_{n=0}^{N} f_{m}(n) e^{-j\left(\Omega T-\frac{2 \pi k}{M}\right) n}=\mathbf{f}_{m}^{T}\left[\mathbf{c}_{k}(\Omega)-j \mathbf{s}_{k}(\Omega)\right],
$$

where $f_{m}(n)$ is the $m$ th digital FIR filter coefficient to be determined, and

$$
\begin{gathered}
\mathbf{f}_{m}=\left[f_{m}(0), f_{m}(1), \ldots, f_{m}(N-1)\right]^{T} \\
\mathbf{c}_{k}(\Omega)=\left[1, \cos \left(\Omega T-\frac{2 \pi k}{M}\right), \ldots, \cos (N-1)\left(\Omega T-\frac{2 \pi k}{M}\right)\right]^{T} \\
\mathbf{s}_{k}(\Omega)=\left[0, \sin \left(\Omega T-\frac{2 \pi k}{M}\right), \ldots, \sin (N-1)\left(\Omega T-\frac{2 \pi k}{M}\right)\right]^{T} .
\end{gathered}
$$

Thus, the real transfer function $T_{k}(j \Omega)$ becomes

$$
\begin{aligned}
T_{k}(j \Omega)=\sum_{m=1}^{M} \mathbf{f}_{m}^{T} \mathbf{r}_{k, m}(\Omega)-j \sum_{m=1}^{M} \mathbf{f}_{m}^{T} \mathbf{i}_{k, m}(\Omega) & =\mathbf{r}_{k}^{T}(\Omega) \mathbf{f}-j \mathbf{i}_{k}^{T}(\Omega) \mathbf{f} \\
& =\operatorname{Re}\left\{T_{k}(j \Omega)\right\}-j \operatorname{Im}\left\{T_{k}(j \Omega)\right\},
\end{aligned}
$$

where

$$
\begin{gathered}
\mathbf{f}=\left[\mathbf{f}_{1}^{T}, \mathbf{f}_{2}^{T}, \ldots, \mathbf{f}_{M}^{T}\right]^{T} \\
\operatorname{Re}\left\{T_{k}(j \Omega)\right\}=\mathbf{r}_{k}^{T}(\Omega) \mathbf{f}, \operatorname{Im}\left\{T_{k}(j \Omega)\right\}=\mathbf{i}_{k}^{T}(\Omega) \mathbf{f} \\
\mathbf{r}_{k}(\Omega)=\left[\mathbf{r}_{k, 1}^{T}(\Omega), \mathbf{r}_{k, 2}^{T}(\Omega), \ldots, \mathbf{r}_{k, M}^{T}(\Omega)\right]^{T}, \quad \mathbf{i}_{k}(\Omega)=\left[\mathbf{i}_{k, 1}^{T}(\Omega), \mathbf{i}_{k, 2}^{T}(\Omega), \ldots, \mathbf{i}_{k, M}^{T}(\Omega)\right]^{T}
\end{gathered}
$$

and

$$
\begin{aligned}
\mathbf{r}_{k, m}(\Omega)= & \frac{1}{M T} \mathbf{c}_{k}(\Omega) \hat{H}_{d l m}\left(e^{j\left(\Omega T-\frac{2 \pi k}{M}\right)-\frac{(m-1)}{M} \pi}\right) H_{a b m}(j \Omega) \\
& \times H\left(j\left(\Omega-\frac{(m-1)}{M T} \pi\right)\right) H_{a l m}\left(j\left(\Omega-\frac{(m-1)}{M T} \pi\right)\right) \\
\mathbf{i}_{k, m}(\Omega)= & \frac{1}{M T} \mathbf{s}_{k}(\Omega) \hat{H}_{d l m}\left(e^{j\left(\Omega T-\frac{2 \pi k}{M}\right)-\frac{(m-1)}{M} \pi}\right) H_{a b m}(j \Omega) \\
& \times H\left(j\left(\Omega-\frac{(m-1)}{M T} \pi\right)\right) H_{a l m}\left(j\left(\Omega-\frac{(m-1)}{M T} \pi\right)\right) .
\end{aligned}
$$

Further, $\operatorname{Re}\{\bullet\}$ and $\operatorname{Im}\{\bullet\}$ denote the real and imaginary parts of a vector or a complex number, respectively. If the analog filters are ideal in the BI-DAC system, an ideal wideband signal $Y(j \Omega)$ will be generated and this ideal signal will be a delayed and scaled version of the input signal, which is called the perfectreconstruction condition. The ideal transfer function of this BI-DAC system is given by

$$
D_{k}(j \Omega)=\left\{\begin{array}{ll}
c e^{-j \Omega M T d}, & k=0 \\
0, & k=1, \ldots, M-1
\end{array}, \quad \Omega \in\left[0, \frac{\pi}{T}\right]\right.
$$


where $d$ and $c$ are the BI-DAC system delay and gain, respectively. The approximation error, can be computed by using (7) and (8) as

$$
\begin{aligned}
e_{k}(\Omega) & =T_{k}(j \Omega)-D_{k}(j \Omega) \\
& =\operatorname{Re}\left\{e_{k}(\Omega)\right\}-j \operatorname{Im}\left\{e_{k}(\Omega)\right\}
\end{aligned}
$$

with

$$
\begin{aligned}
& \operatorname{Re}\left\{e_{k}(\Omega)\right\}=\mathbf{r}_{k}^{T}(\Omega) \mathbf{f}-R_{k}(\Omega) \\
& \operatorname{Im}\left\{e_{k}(\Omega)\right\}=\mathbf{i}_{k}^{T}(\Omega) \mathbf{f}-I_{k}(\Omega) .
\end{aligned}
$$

where

$$
R_{k}(\Omega)=\operatorname{Re}\left\{D_{k}(\Omega)\right\}, \quad I_{k}(\Omega)=\operatorname{Im}\left\{D_{k}(\Omega)\right\} .
$$

To approximate the perfect-reconstruction in the minimax sense, the minimax design can be formulated as follows:

$$
\begin{array}{ll}
\operatorname{minimize} & \delta \\
\text { subject to } & W_{k}(\Omega)\left|e_{k}(\Omega)\right| \leq \delta, \quad \Omega \in \Omega_{I}, \quad k=0, \ldots, M-1,
\end{array}
$$

where $W_{k}(\Omega)$ is a positive weighting parameter specifying the relative importance between the distortion and aliasing errors, and $\Omega_{I}$ is the first Nyquist zone of the BI-DAC system. It can be known from (10) that the real part approximation error $\operatorname{Re}\left\{e_{k}(\Omega)\right\}$ and the imaginary part approximation error $\operatorname{Im}\left\{e_{k}(\Omega)\right\}$ are all the function of the digital FIR filter coefficients vector $\mathbf{f}$. Consequently, we can reformulate the minimax design (11) as

$$
\begin{array}{ll}
\operatorname{minimize} & \delta_{R}+\delta_{I} \\
\text { subject to } & W_{k}(\Omega)\left|\operatorname{Re}\left\{e_{k}(\Omega)\right\}\right| \leq \delta_{R} \\
& W_{k}(\Omega)\left|\operatorname{Im}\left\{e_{k}(\Omega)\right\}\right| \leq \delta_{I}
\end{array}
$$

Clearly, the minimax problem in (12) can be solved by using LP. The constraints in (12) can be rewritten as

$$
\begin{aligned}
& -\delta_{R} \leq W_{k}(\Omega) \operatorname{Re}\left\{e_{k}(\Omega)\right\} \leq \delta_{R} \\
& -\delta_{I} \leq W_{k}(\Omega) \operatorname{Im}\left\{e_{k}(\Omega)\right\} \leq \delta_{I}
\end{aligned}
$$

i.e.,

$$
\begin{aligned}
& -\delta_{R} \leq W_{k}(\Omega)\left(\mathbf{r}_{k}^{T}(\Omega) \mathbf{f}-R_{k}(\Omega)\right) \leq \delta_{R} \\
& -\delta_{I} \leq W_{k}(\Omega)\left(\mathbf{i}_{k}^{T}(\Omega) \mathbf{f}-I_{k}(\Omega)\right) \leq \delta_{I}
\end{aligned}
$$

or equivalently

$$
\left\{\begin{array}{l}
-\delta_{R}-W_{k}(\Omega) \mathbf{r}_{k}^{T}(\Omega) \mathbf{f} \leq-W_{k}(\Omega) R_{k}(\Omega) \\
-\delta_{R}+W_{k}(\Omega) \mathbf{r}_{k}^{T}(\Omega) \mathbf{f} \leq W_{k}(\Omega) R_{k}(\Omega)
\end{array}\right.
$$

and

$$
\left\{\begin{array}{l}
-\delta_{I}-W_{k}(\Omega) \mathbf{i}_{k}^{T}(\Omega) \mathbf{f} \leq-W_{k}(\Omega) I_{k}(\Omega) \\
-\delta_{I}+W_{k}(\Omega) \mathbf{i}_{k}^{T}(\Omega) \mathbf{f} \leq W_{k}(\Omega) I_{k}(\Omega)
\end{array}\right.
$$

In the frequency band $\Omega_{I}$, the frequency variable $\Omega$ is discretized over a dense set of frequency points $\left\{\Omega_{i}, 1 \leq i \leq P_{L}\right\} . P_{L}$ is the total number of these frequency points. $\Omega_{i}$ is evenly distributed in $\Omega_{I}$. Using the one-to-one mapping $\Omega_{i} \rightarrow i$, we get the one-to-one mappings 


$$
\begin{gathered}
W_{k}\left(\Omega_{i}\right) \mathbf{r}_{k}^{T}\left(\Omega_{i}\right) \rightarrow \mathbf{W}_{k}^{R}(i,:) \\
W_{k}\left(\Omega_{i}\right) R_{k}\left(\Omega_{i}\right) \rightarrow d_{k}^{R}(i)
\end{gathered}
$$

and

$$
\begin{gathered}
W_{k}\left(\Omega_{i}\right) \mathbf{i}_{k}^{T}\left(\Omega_{i}\right) \rightarrow \mathbf{W}_{k}^{I}(i,:) \\
W_{k}\left(\Omega_{i}\right) I_{k}\left(\Omega_{i}\right) \rightarrow d_{k}^{I}(i) .
\end{gathered}
$$

As a result, (12) is cast to the following LP problem

$$
\begin{array}{ll}
\text { minimize } & \mathbf{c}^{T} \mathbf{x}_{1}+\mathbf{c}^{T} \mathbf{x}_{2} \\
\text { subject to } & \mathbf{A}_{k}^{R} \mathbf{x}_{1} \leq \tilde{\mathbf{d}}_{k}^{R} \\
& \mathbf{A}_{k}^{I} \mathbf{x}_{2} \leq \tilde{\mathbf{d}}_{k}^{I},
\end{array}
$$

where

$$
\begin{gathered}
\mathbf{c}=\left[1, \mathbf{O}_{1 \times N M}\right]^{T}, \quad \mathbf{x}_{1}=\left[\delta_{R}, \mathbf{f}^{T}\right]^{T}, \quad \mathbf{x}_{2}=\left[\delta_{I}, \mathbf{f}^{T}\right]^{T} \\
\mathbf{A}_{k}^{R}=\left[\begin{array}{cc}
-\mathbf{I}_{P_{L} \times 1} & -\mathbf{W}_{k}^{R} \\
-\mathbf{I}_{P_{L} \times 1} & \mathbf{W}_{k}^{R}
\end{array}\right], \quad \mathbf{A}_{k}^{I}=\left[\begin{array}{cc}
-\mathbf{I}_{P_{L} \times 1} & -\mathbf{W}_{k}^{I} \\
-\mathbf{I}_{P_{L} \times 1} & \mathbf{W}_{k}^{I}
\end{array}\right] \\
\tilde{\mathbf{d}}_{k}^{R}=\left[\begin{array}{c}
-\mathbf{d}_{k}^{R} \\
\mathbf{d}_{k}^{R}
\end{array}\right], \quad \tilde{\mathbf{d}}_{k}^{I}=\left[\begin{array}{c}
-\mathbf{d}_{k}^{I} \\
\mathbf{d}_{k}^{I}
\end{array}\right] \\
\mathbf{d}_{k}^{R}=\left[d_{k}^{R}(1), d_{k}^{R}(2), \ldots, d_{k}^{R}\left(P_{L}\right)\right]^{T}, \quad \mathbf{d}_{k}^{I}=\left[d_{k}^{I}(1), d_{k}^{I}(2), \ldots, d_{k}^{I}\left(P_{L}\right)\right]^{T} .
\end{gathered}
$$

Additionally, $\mathbf{O}_{1 \times N M}$ and $\mathbf{I}_{P_{L} \times 1}$ denote, respectively, a 1-by-NM zero vector and a $P_{L}$-by-1 one vector. Here, SeDuMi can be used to solve the LP problem in (13), which applies a variant of the primaldual interior-point method called centeringpredictor-corrector method [13].

The digital FIR filter length $N$ is a significant parameter that determines the BIDAC system performance. As we know, when $N$ is longer, the distortion error is smaller, but the aliasing error attenuation is larger and the system complexity also increases. As mentioned in Section 1, the performance of the DAC is evaluated by SFDR. The relationship between the desired SFDR and the BI-DAC system resolution is described as $S F D R(\mathrm{dBc})=6.02 \times S F D R_{\text {bits }}$, where $S F D R_{\text {bits }}$ denotes the effective number of bits [14]. For a given resolution $S F D R_{b i t s}$ in the BI-DAC system, the digital FIR filter length $N$, used in this proposed design method, is chosen in a way that decreases the distortion error to nearly the ideal of $0 \mathrm{~dB}$, and the aliasing errors are reduced to a level lower than the desired SFDR.

\subsection{Computational complexity}

The main aim of this paper is to reduce the computational complexity of the digital FIR filters optimal design in the BI-DAC system. For instance, if we use the optimization design method in [10], the computational complexity is $O\left(N_{D 1}\left(N M\left(M P_{L}\right)^{3}+\left(1+M P_{L}\right)(N M)^{2}\right)\right)$, where $N_{D 1}$ is the number of iterations of this design method.

In contrast to the optimization design method in [10], the computational complexity of our proposed design is derived as follows:

1) Obtaining the matrix $\mathbf{A}_{k}^{R} \mathbf{x}_{1}$ in (13) requires $O\left(2 P_{L}(N M+1)\right.$ ).

2) Obtaining the matrix $\mathbf{A}_{k}^{I} \mathbf{x}_{2}$ in (13) requires $O\left(2 P_{L}(N M+1)\right)$. 
Therefore, the computational complexity of the minimax design using LP is $O\left(4 P_{L} \times N_{D 2}(N M+1)\right)$, where $N_{D 2}$ is the number of iterations of our proposed design method. It can be seen that the computational complexity of the minimax design using LP is lower than that of the optimization design method in [10].

\section{Design examples and simulation results}

In this section, numerical design examples are conducted to evaluate and analyze the effectiveness and the computational complexity of our proposed design method.

\subsection{Effectiveness analysis}

To evaluate the effectiveness of our proposed design method, we designed a fourchannel 10-bit BI-DAC system with a system sampling rate $f_{s}=\frac{1}{T}$. Unless otherwise specified, in this design example, the BI-DAC system delay $d$, the system gain $c$, the number of the frequency sample points, and the order of the digital FIR filters are set to 25, 1, 80, and 50, respectively. The LP problem was solved using the SeDuMi Matlab toolbox [13]. The effective number of bits of the BI-DAC system can be taken any values, it is only used to calculate the desired SFDR and there is no any influence on the simulation result. As mentioned in Section 3.2, the desired SFDR of this BI-DAC system is $10 \times 6.02=60.2 \mathrm{~dB}$. Consequently, the aliasing errors should be lower than $-60.2 \mathrm{~dB}$ in this design example. Second-order Butterworth filters were chosen as the analog analysis filters. The digital FIR filters were designed using LP under the minimax criteria. The positive weighting parameter $W_{k}(\Omega)$ for $k=0,1,2,3$ all were set to 1 in the BI-DAC system output frequency band $\left[0, \frac{\pi}{T}\right]$. As described in Section 1 , the optimization design method in [10] is also suitable for the BI-DAC system so that it is carried out for comparison.

The frequency responses of the optimization design method in [10] and the minimax design using LP are shown in Fig. 2. It can be seen from Fig. 2(a) that, the maximum distortion error of the optimization design method in [10] was around $0.05 \mathrm{~dB}$, and the minimax design using LP had a lower distortion error which was around $0.02 \mathrm{~dB}$. From Fig. 2(b), we can see that, the minimum and maximum aliasing errors of the minimax design using LP were $-61 \mathrm{~dB}$ and $-100 \mathrm{~dB}$, respectively. So it satisfied the desired SFDR. In contrast, the minimum and maximum aliasing errors of the optimization design method in [10] were, respectively, $-50 \mathrm{~dB}$ and $-85 \mathrm{~dB}$ so that the desired SFDR could not be satisfied. Consequently, the minimax design using LP can more effectively cancel the aliasing errors than the optimization design method in [10].

\subsection{Computational complexity analysis}

As described in Section 3.3, we compared the computational complexity between the optimization design method in [10] and the minimax design using LP using the following parameter: $P_{L}=80, N_{D 1}=8$ and $N_{D 2}=5$.

Fig. 3 shows, respectively, the computational complexity comparisons of these two design methods versus the digital FIR filters length when the number of the sub-channels $M=4$, and the computational complexity comparisons of these two 

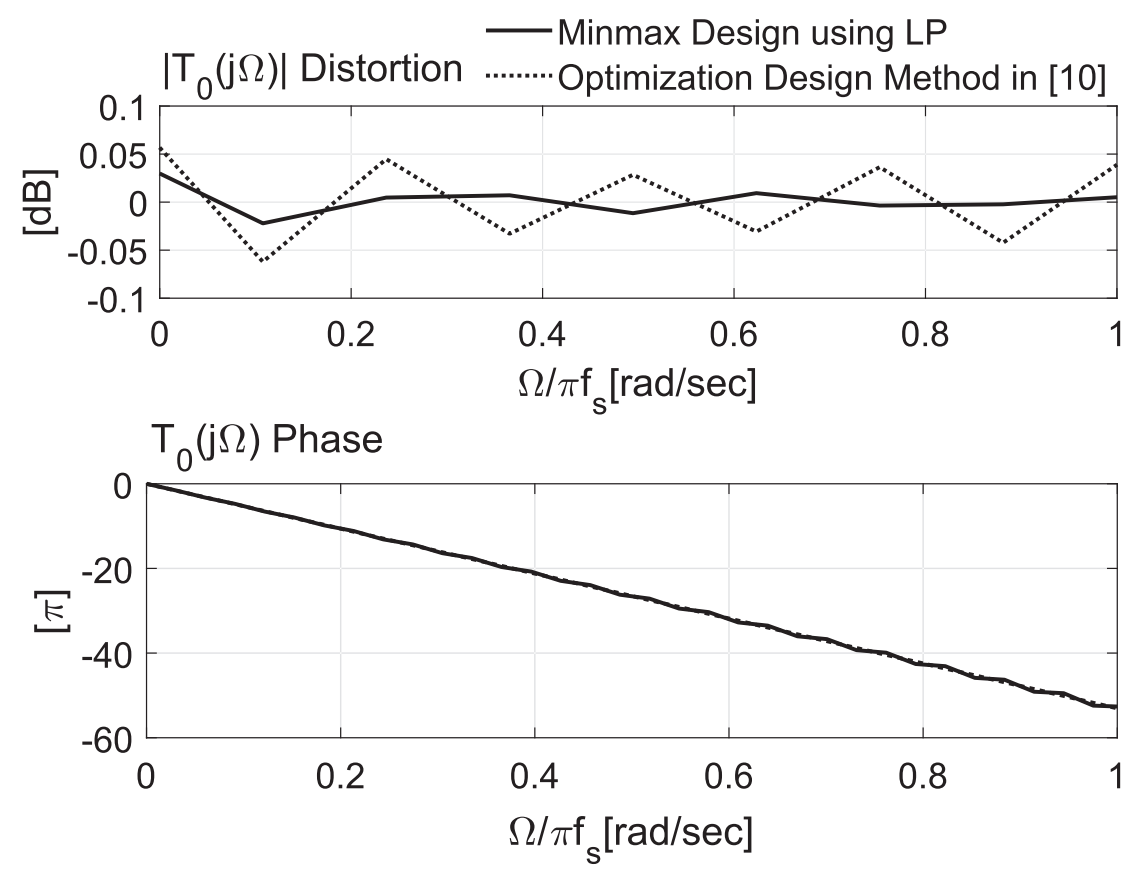

(a)
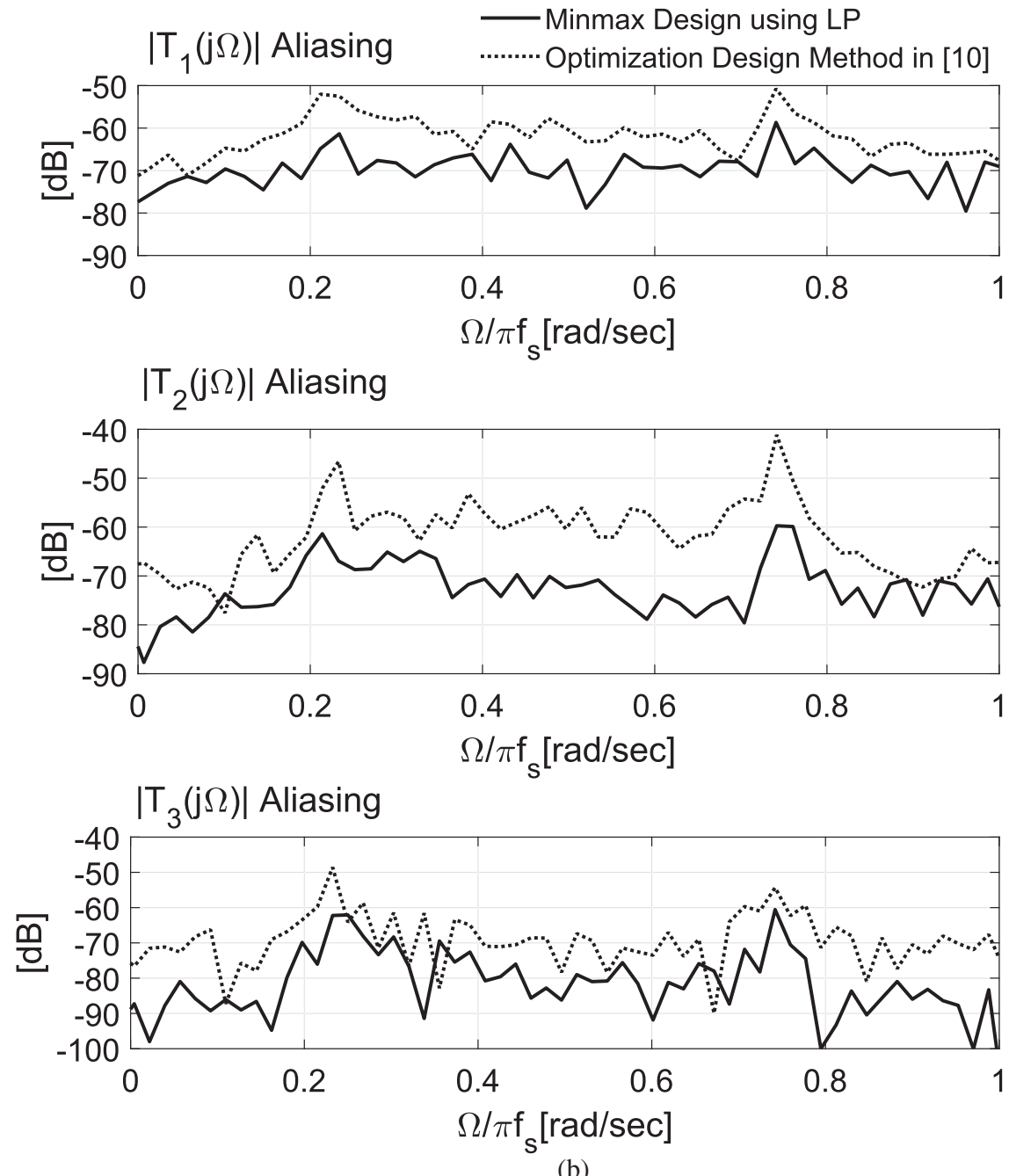

Fig. 2. Optimization design method in [10] and Minimax design using LP: (a) Frequency responses of the distortion function, (b) Frequency responses of the aliasing functions. 


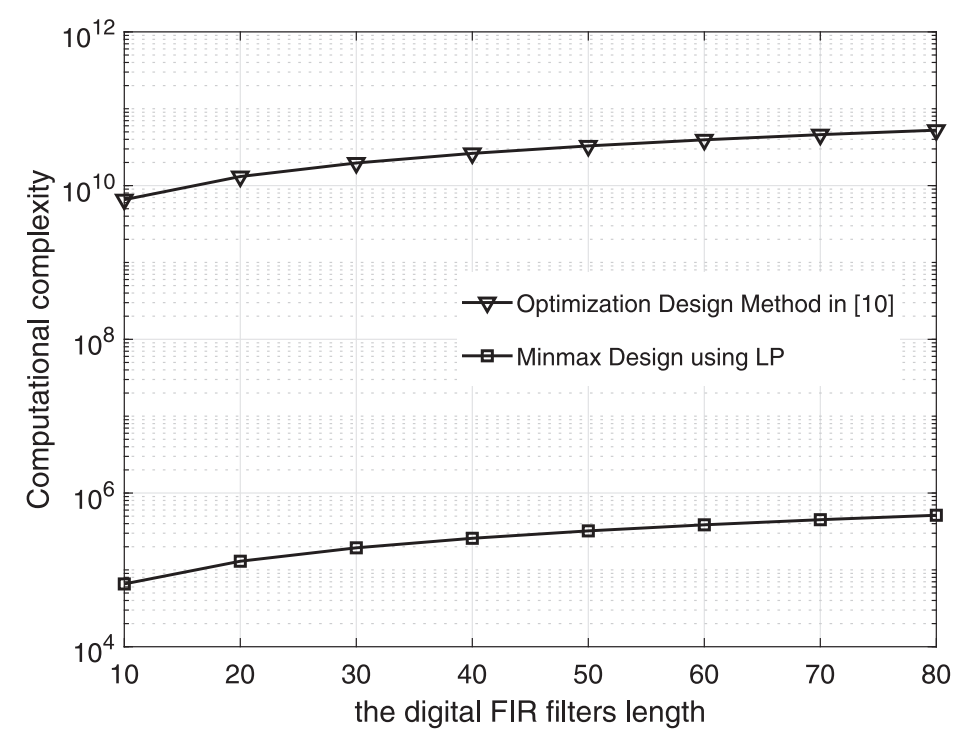

(a)

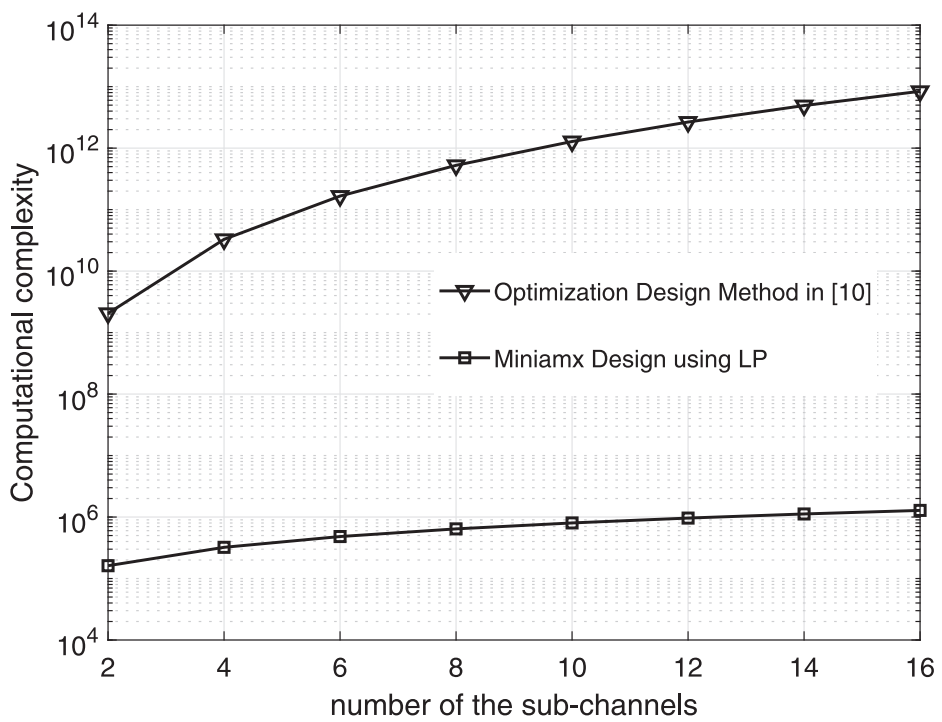

(b)

Fig. 3. Comparisons of the computational complexity between the optimization design method in [10] and Minimax design using LP: (a) Complexity versus the digital FIR filters length when the number of the sub-channels $M=4$, (b) Complexity versus the number of the sub-channels when the digital FIR filters length $N=50$.

design methods versus the number of the sub-channels when the digital FIR filters length $N=50$. From Fig. 3(a) and (b), we found that the computational complexity of these two design methods raised as the digital FIR filters length or the number of the sun-channels increased. Further, the minimax design using LP had much lower computational complexity than the optimization design method in [10], which is the same as the description in Section 3.3. Therefore, comparing with the optimization design method in [10], our proposed design method can achieving the goal for reducing the computational complexity of the digital FIR filters optimal design in the BI-DAC system. 


\section{Conclusion}

In this paper, we investigated the principle of the BI-DAC system and deduced the real transfer function of this system. To cancel the aliasing errors caused by the non-ideal analog filters performance in the BI-DAC system, the minimax design of the digital FIR filters design using LP was proposed. As an optimization problem, LP can be used to find the global optimal solution. Theoretical analysis and simulation results demonstrated that the minimax design using LP had more effective aliasing errors cancellation and much lower computational complexity than the optimization design method in [10]. Therefore, the mimimax design using LP is more applicable to the case with lower aliasing errors and computational complexity.

In the future work, we will explore how to formulate the problem of designing the digital FIR filters as another optimization problems such as second-order cone programming (SOCP) which is one of the convex optimization problems.

\section{Acknowledgments}

This work was supported in part by the National Natural Science Foundation of China under Grant 61671114. 